\title{
Lymphocyte mitogenesis, immunoglobulin and complement levels in depressed patients and normal controls
}

\author{
Z. Kronfol', J.D. House ${ }^{2}$ \\ 'Department of Psychiatry, University of Michigan \\ Medical Center, Ann Arbor, Michigan, ${ }^{2}$ Department of \\ Family Practice, University of lowa College of \\ Medicine, lowa City, lowa, USA
}

\begin{abstract}
The central nervous system and the immune system are closely related. Psychiatric illness is often associated with a dysregulation of the immune response. In an attempt to expand on previously reported immune abnormalities in patients with depressive illness, we compared several immune measures in a group of hospitalized depressed patients and healthy normal controls. Depressed patients had significantly higher percentages of circulating neutrophils, significantly lower percentages of circulating lymphocytes and significantly lower in vitro lymphocyte responses to mitogenic stimulation than normal controls. Basal plasma cortisol and circulating levels of the complement components $\mathrm{C} 3$ and $\mathrm{C} 4$ were also higher in the depressed group. We also found a significant association between cortisol values and the traffic of leukocytes on the one hand, and complement levels and the lymphocyte mitogenic activities on the other. These findings expand previously reported evidence of immune abnormalities in depressive illness and provide a partial explanation for some of these findings.
\end{abstract}

Received November 17, 1988; accepted for publication March 31, 1989

There is now ample evidence of interactions between the central nervous system and the immune system (1). Both stress $(2,3)$ and psychiatric illness $(4,5)$ have been associated with impairment in immune function. We have earlier reported a reduction in mitogen-induced lymphocyte proliferation in patients with depressive illness (6). Our findings have been replicated by several investigators $(7,8)$. Most of the studies so far have examined only 1 or 2 aspects of immune function at a time in a small number of patients. In this study, we compared several aspects of immune function (leukocyte traffic, lymphocyte subsets, mitogen responses and immunoglobulin levels) in a relatively large population of hospitalized depressed patients and normal controls. Because cortisol has often been suspected of playing a major role as a link between the central nervous system and the immune system (9), we also examined the relationship between plasma cortisol values and the different immune function tests. Serum complement is also an important mediator of immune function and is often increased in certain types of psychological stress as an "acute phase reactant" (10). We therefore compared circulating levels of 2 complement components $\mathrm{C} 3$ and $\mathrm{C} 4$ in depressed patients and normal controls and examined the relationship between these proteins and different immune variables.

\section{Material and methods Subjects}

Subjects for the study were recruited from consecutive admissions to the University of Iowa Psychiatric 
hospital. Patients were included in the study if they met DSM-III (11) criteria for major depressive disorder, were free of major medical or neurological disorders known to interfere with immune regulation such as infection or cancer, free of alcoholism and drug abuse, and free of medication known to affect immune function. Psychoactive drugs as such were not, however, considered exclusion criteria. Forty patients, 18 male, 22 female, were included in the study. The mean $( \pm S D)$ age of the depressed patients was $42.6 \pm 16.4$ years. Their age range was 21-76 years. They scored a mean $( \pm S D)$ of 26.2 \pm 9.8 on the Carroll Rating Scale for Depression. Blood for immune and endocrine investigation was collected as soon as possible following the patient's admission to the hospital, usually within 10 days following admission. Normal controls were healthy volunteers who were free of medical and psychiatric illness and were not taking medication at the time of the study. The mean $( \pm S D)$ age of the control group was $31.3 \pm 9.0$ years (range 20-58). Nineteen subjects were male; 18 were female. They scored a mean ( \pm SD) of $1.7 \pm 1.5$ on the Carroll Rating Scale for Depression. Both patients and controls who participated in the study had all given informed consent.

\section{Methods}

Blood collection. Twenty milliliters of blood were drawn from each subject between 8:00 a.m. and 9:00 a.m. in a heparinized tube. Whenever possible, blood samples from patients and controls were drawn and processed together on the same day to control for day-to-day variation in the assay. A total white blood cell (WBC) count and differential was performed on each sample using standard procedures. The blood was then centrifuged over FicollHypaque and the lymphocytes separated for culture or surface marker analyses (see below). The plasma was frozen and stored at $-70^{\circ} \mathrm{C}$ for cortisol, immunoglobulin and complement level analysis (see below)

Lymphocyte mitogenic stimulation. Lymphocyte cultures were established in triplicate as previously described (6). To each well, one of the following mitogens was added: no mitogen for background transformation (blank); phytohemagglutinin-P (PHA), $2.5 \mu \mathrm{g} / \mathrm{ml}$; concanavalin A (Con A), 10 $\mu \mathrm{g} / \mathrm{ml}$; and pokeweed mitogen (PWM), $2.5 \mu \mathrm{g} / \mathrm{ml}$. These concentrations were chosen because they were associated with maximum stimulation in our laboratory. The cultures were kept for $\mathbf{4}$ days prior to addition of tritiated thymidine and harvesting. Tritiated thymidine uptake was then measured with a scintillation counter. Mean scintillation counts per minute (cpm) were calculated for each mitogen, and the 2 groups were compared.

Surface marker analysis. For the surface marker analyses, lymphocytes from each subject were washed several times and incubated separately with one of the following commercially available monoclonal antibodies (Coulter Immunology, Hialeah, FL, USA): CD20 for total B lymphocytes; CD2 for total $\mathrm{T}$ lymphocytes; $\mathrm{CD} 4$ for $\mathrm{T}$ helper/inducer cells; and CD8 for T suppressor/cytoxic cells. The cells were then washed again and incubated with a fluorescein-conjugated second antibody (GAMFITC, Coulter Immunology). The frequency (percentage) of each cell type was then determined with fluorescent microscopy.

Immunoglobulin, complement and cortisol levels. Immunoglobulin and complement levels of plasma samples were measured using a standard radial immunodiffusion kit (Nor-Partigen, CalbiochemBehring, La Jolla, CA, USA). Plasma cortisol was measured using a standard radioimmunoassay procedure.

Statistical analysis. Because the difference in age distribution between depressed patients and normal controls was statistically significant $(P<0.001)$, comparisons on all other dependent variables between the 2 groups were done using analysis of covariance (ANCOVA), with age as a covariate. A difference between the 2 groups was considered statistically significant when $P<0.05$. For correlations between the endocrine and immune variables, we calculated Pearson's correlation coefficient $(r)$. All levels of significance were two-tailed.

\section{Results}

Results of the hematological variables in depressed patients and normal controls are shown in Table 1. There were no significant differences in hematocrit between depressed patients and normal controls. WBC counts, however, were significantly higher in the depressed groups $(P<0.01)$. The percentage of neutrophils was also significantly higher among depressed patients $(P<0.001)$. The percentages of 
Table 1

Hematological variables

\begin{tabular}{lcc}
\hline & Depression & Normal controls \\
\hline Hematocrit (\%) & $45.1 \pm 4.1$ & $43.8 \pm 3.5$ \\
WBC count (per mm $\mathrm{mm}^{3}$ ) & $8565 \pm 3016$ & $6787 \pm 1799^{* *}$ \\
Neutrophil (\%) & $64.1 \pm 11.5$ & $53.1 \pm 10.0^{* * *}$ \\
Lymphocyte (\%) & $29.5 \pm 10.7$ & $38.5 \pm 9.7^{* *}$ \\
Monocyte (\%) & $3.6 \pm 1.9$ & $5.0 \pm 2.2^{*}$ \\
Eosinophils (\%) & $2.1 \pm 1.7$ & $2.6 \pm 1.3$ \\
Basophils (\%) & $0.82 \pm 0.81$ & $0.73 \pm 0.84$ \\
\hline
\end{tabular}

Means $\pm \mathrm{SD} ;{ }^{*} P<0.05 ;{ }^{* *} P<0.01 ; * * P<0.001$.

Table 2

Lymphocyte subset populations*

\begin{tabular}{lcc}
\hline & Depression & Normal controls \\
\hline Total lymphocytes* & $2490 \pm 1071$ & $2540 \pm 629$ \\
B cells & $231 \pm 103$ & $241 \pm 154$ \\
T cells & $1574 \pm 811$ & $1709 \pm 553$ \\
T helper/inducer cells & $937 \pm 499$ & $998 \pm 322$ \\
T suppressor/cytotoxic cells & $586 \pm 362$ & $647 \pm 238$ \\
T helpers/T suppressors & $1.79 \pm 0.84$ & $1.63 \pm 0.48$ \\
\hline
\end{tabular}

*Results are expressed as number of cells per $\mathrm{mm}^{3}$; means $\pm \mathrm{SD}$.

lymphocytes and monocytes, on the other hand, were significantly lower in the depressed group $(P<0.01$ and $P<0.05$, respectively). No significant differences in the percentages of eosinophils or basophils emerged between the 2 groups.

Table 2 summarizes the results of the different lymphocyte subsets between the depressed patients and the normal controls. There were no significant differences in either the absolute numbers of lymphocytes or the percentage of the different lymphocyte subsets between the 2 groups. Similarly, the ratio of helper-to-suppressor $T$ lymphocytes (CD4/ CD8) was not significantly different between depressed patients and normal controls.

Results of the in vitro lymphocyte response to mitogen stimulation are shown in Table 3. There were no significant differences in the unstimulated lymphocyte responses (blank). Lymphocytic responses to the mitogens PHA, Con A and PWM were lower in the depressed patients compared with normal controls. These differences were statistically significant for Con $A(P<0.001)$ and PWM $(P<0.05)$ but not for PHA.

Results of immunoglobulin, complement and cortisol values are shown in Table 4. Immunoglobulin $\mathbf{M}$ was slightly higher among depressed patients
$(P<0.05)$ than among normal controls. The immunoglobulins IgG and IgA were not significantly different among the groups. The complement components $\mathrm{C} 3$ and $\mathrm{C} 4$ were higher among the depressed patients than among the normal controls $(P<0.01$ and $P<0.05$, respectively). Cortisol values were also higher among the depressed group $(P<0.001)$.

Table 5 summarizes correlations between plasma cortisol values and selected immune measures. There was a positive correlation between plasma

Table 3

Lymphocyte mitogenic responses

\begin{tabular}{lcc}
\hline & Depression & Normal controls \\
\hline Blank & $1,056 \pm 859$ & $940 \pm 702$ \\
PHA $(2.5 \mu \mathrm{g} / \mathrm{ml})$ & $146,253 \pm 60,546$ & $177,773 \pm 66,867$ \\
& $(218.5 \pm 175.8)$ & $(244.5 \pm 122.4)$ \\
Con A $(10 \mu \mathrm{g} / \mathrm{ml})$ & $88,815 \pm 47,037$ & $151,067 \pm 84,373^{* * *}$ \\
& $(121.8 \pm 106.4)$ & $(185.2 \pm 100.0)$ \\
PWM $(2.5 \mu \mathrm{g} / \mathrm{ml})$ & $77,716 \pm 28,820$ & $109,275 \pm 62,559^{*}$ \\
& $(110.9 \pm 85.8)$ & $(137.8 \pm 68.7)$ \\
\hline
\end{tabular}

Results are expressed as scintillation cpm: means \pm SD. The ratio of stimulated to unstimulated lymphocytes for each of the mitogens is shown in parentheses. ${ }^{*} P<0.05,{ }^{* * *} P<0.001$.

Table 4

Immunoglobulins, complement components and plasma cortisol levels

\begin{tabular}{lcc}
\hline & Depression & Normal controls \\
\hline $\operatorname{IgG}(\mathrm{g} / \mathrm{l})$ & $12.34 \pm 3.11$ & $12.32 \pm 3.22$ \\
$\operatorname{IgM}(\mathrm{g} / \mathrm{l})$ & $1.70 \pm 0.75$ & $1.66 \pm 0.76^{* \mathrm{a}}$ \\
$\mathrm{IgA}(\mathrm{g} / \mathrm{l})$ & $2.0 \pm 0.79$ & $1.88 \pm 0.83$ \\
$\mathrm{C} 3(\mathrm{~g} / \mathrm{l})$ & $0.87 \pm 0.15$ & $0.76 \pm 0.10^{* *}$ \\
$\mathrm{C} 4(\mathrm{~g} / \mathrm{l})$ & $0.41 \pm 0.14$ & $0.36 \pm 0.87^{*}$ \\
$\mathrm{Cortisol}(\mu \mathrm{g} / \mathrm{l})$ & $161 \pm 42$ & $108 \pm 52^{* * *}$ \\
\hline
\end{tabular}

Means \pm SD. ${ }^{*} P<0.05 ;{ }^{*} P<0.01 ;{ }^{* *} P<0.001$. $^{\text {a }}$ The difference in IgM values, although small, is statistically significant because of a strong negative correlation between IgM values and age. Statistical analyses were done with ANCOVA with age as a covariate.

Table 5

Correlations between basal cortisol values and selected immune measures

\begin{tabular}{lc}
\hline Immune measure & $r$ \\
\hline WBC count & 0.10 \\
Neutrophil (\%) & $0.37^{* *}$ \\
Lymphocyte (\%) & $-0.35^{* *}$ \\
PHA & -0.04 \\
Con A & -0.04 \\
PWM & -0.07 \\
\hline
\end{tabular}

** $P<0.01$ 
Table 6

Correlations between complement components $\mathrm{C}_{4}$ and selected immune measures

\begin{tabular}{lc}
\hline Immune measure & \multicolumn{1}{c}{} \\
\hline WBC count & $0.25^{*}$ \\
Neutrophil $(\%)$ & 0.10 \\
Lymphocyte $(\%)$ & -0.12 \\
PHA & $-0.37^{* *}$ \\
Con A & $-0.33^{* *}$ \\
PWM & $-0.26^{*}$ \\
\hline
\end{tabular}

${ }^{*} P<0.05 ;{ }^{* *} P<0.01$.

cortisol values and the percentage of neutrophils $(P<0.01)$, and a negative correlation between plasma cortisol values and the percentage of lymphocytes $(P<0.01)$. There were no significant correlations between plasma cortisol values and lymphocyte subsets, lymphocyte mitogenic responses, immunoglobulin or complement levels.

Relations between complement component $\mathrm{C} 4$ and selected immune variables are shown in Table 6. There was a positive correlation between $\mathrm{C} 4$ and WBC counts $(r=0.25, P<0.05)$. There were also negative correlations between $\mathrm{C} 4$ and the lymphocytic responses to all 3 mitogens, PHA $(r=-0.37 ; P<0.01)$, Con A $(r=-0.33 ; P<0.01)$ and PWM $(r=-0.26 ; P<0.05)$. These was also a strong positive correlation between $\mathrm{C} 3$ and $\mathrm{C} 4$ $(r=0.47 ; P<0.001)$.

\section{Discussion}

This study was undertaken to further explore possible abnormalities in immune functioning in depressed patients compared with normal controls. The results indicate significant differences in the number and traffic of leukocytes, a significant reduction in lymphocyte responsiveness to mitogenic stimulation, and a significant increase in plasma levels of IgM, complement components C3 and C4 and cortisol values in the depressive group. Results regarding the traffic of leukocytes that consist of an increase in the percentage of neutrophils and a decrease in the percentage of lymphocytes and monocytes confirm our previously reported findings in an unmedicated group of depressive patients (12). Further, there were no significant differences in the traffic of leukocytes between unmedicated depressed patients $(n=10)$ and those who were receiving psychotropic medications $(n=30)$. These findings, as we speculated earlier, are consistent with the expected hematological effects of higher cortisol values in the depressed group: an increase in the percentage of neutrophils and a decrease in the percentage of lymphocytes. The significant correlations between plasma cortisol values and neutrophil and lymphocyte percentages (Table 5) provide further confirmation of a probable relationship between plasma cortisol values and the traffic of leukocytes.

The distribution of lymphocyte subsets, on the other hand, did not differ significantly between depressed patients and normal controls. These results are consistent with previous reports in the literature (13), although some data, like our own, did show a tendency for $\mathrm{CD} 4 / \mathrm{CD} 8$ to be higher among depressed patients (14). It should, however, be noted that we investigated, with the use of monoclonal antibodies, only 4 different markers on the surface of the lymphocytes. Technology is expanding rapidly in this field and there are presently more than 20 different markers on the surface of the lymphocyte that can be quantified and compared. A more comprehensive evaluation of lymphocyte surface markers identifying newer lymphocyte subsets and using flow cytometry technology is presently underway.

Results concerning the lymphocyte responses to mitogenic stimulation confirm our previous findings (6), which have since been replicated by several investigators $(7,8)$. It should, however, be noted that uniike our previous report, which described lymphocyte mitogenic responses in depressed patients who were drug-free for at least 2 weeks, the present data were obtained on depressed patients, the majority of whom were receiving psychotropic medications. Still, because of the possibility that psychotropic medications may affect lymphocyte mitogenic activity, we compared lymphocytic responses in the unmedicated depressed patients $(n=10)$ with those who were receiving psychotropic medications $(n=30)$. There were no significant differences in the responses to any of the mitogens used. These results further confirm the reduction in lymphocyte mitogenic activity among depressed patients and suggest that psychotropic medications alone cannot explain the differences observed between patients and controls.

Results of the plasma concentrations of the 
immunoglobulins $\operatorname{IgG}, \operatorname{IgM}$ and $\operatorname{IgA}$ showed a mild increase in the depressed group. This increase was statistically significant for IgM, but not for IgG or IgA. Again, these results are consistent with most published reports $(15,16)$. The findings of significant increases in the complement components $\mathrm{C} 3$ and $\mathrm{C} 4$ were somewhat unexpected. Only one group of investigators have reported an elevation of $\mathrm{C} 4$, but not C3, in patients with bipolar psychosis (15). Our results confirm and expand these findings. An increase in $\mathrm{C} 3$ and $\mathrm{C} 4$, together with elevations in WBC counts and the levels of circulating immunoglobulins, suggest an acute phase reaction such as the result of viral or antigenic stimulation. The significant increase in plasma cortisol values in depressed patients is consistent with previous reports of hypercortisolemia in depressive illness (17), and may also accompany the acute phase reaction. Again, there were no significant differences between medicated and unmedicated depressed patients in immunoglobulin, complement or cortisol values.

Because an increase in complement components has been associated with a reduction of the immune response (18), we examined the relationship between plasma $\mathrm{C} 4$ values and the different immune variables (Table 6). We found a significant positive correlation between $\mathrm{C} 4$ and WBC counts and significant negative correlations between $\mathrm{C} 4$ and each of the 3 mitogens used. These results are suggestive of an association between the elevated complement components and the reduction in lymphocyte mitogenesis.

In summary, we compared a group of hospitalized depressed patients and a group of healthy, normal controls on several immunological tests. Depressed patients had higher neutrophil percentages and lower lymphocyte percentages than normal controls. These findings may be explained on the basis of higher cortisol values among the depressed group. Depressed patients also had lower lymphocyte mitogenic responses than the control group. These findings cannot be explained by the higher cortisol values, but may possibly be related to the higher complement levels in the depressed group. Further research on the interaction between hormones and immunity in depression is warranted.

\section{Acknowledgements}

This work was supported in part by the Nellie Ball Trust Fund, the Department of Psychiatry of the University of lowa College of Medicine, and NIMH Grant MH 42988. We thank Janice Quinn for technical assistance and Cathy Schleif for typing the manuscript. We also thank Dr. Stanley Schwartz, Director, Clinical Immunology, and Dr. Madhavan Nair, Laboratory Director, Psychoimmunology, for reviewing the manuscript.

\section{References}

1. Ader R. Psychoneuroimmunology. New York: Academic Press, 1981.

2. Jemmott JB, Locke SE. Psychosocial factors, immunologic medication, and human susceptibility to infectious illness: how much do we know? Psychol Bull 1984: 95:78-108.

3. Kiecolt-Glaser JK, Garner W, Speicher C, Penn GM, Holliday J, Glaser R. Psychosocial modifiers of immunocompetence in medical students. Psychosom Med 1984:46:7-14.

4. DeLisi LE, King AC, Targum S. Serum immunoglobulin concentrations in patients admitted to an acute Psychiatric inpatient service. Br J Psychiatry 1984:145:661-665.

5. Calabrese J, Kling M, Gold PW. Alterations in immunocompetence during strress, bereavement, and depression: focuses on neuroendocrine regulation. Am J Psychiatry 1987: 144:1123-1134.

6. Kronfol Z, Silva J, Greden J, Dembinski S, Gardner R, Caroll B. Impaired lymphocyte function in depressive illness. Life Sci 1983:33:241-247.

7. Schleifer SJ, Keller SE, Meyerson AT, Raskin MJ, Davis KL, Stein M. Lymphocyte function in major depressive disorder. Arch Gen Psychiatry 1984:41:484-486.

8. Calabrese JR, Skwerer RG, Barna B, et al. Depression, immunocompetence, and prostaglandins of the E series. Psychiatry Res 1986:17:41-47.

9. Kronfol Z, House JD, Silva J, Greden J, Caroll B. Depression, urinary free cortisol excretion and lymphocyte function. $\mathrm{Br} \mathbf{J}$ Psychiatry 1986: 148:70-73.

10. Alexander JW, Good RA. Fundamentals of clinical immunology. Philadelphia: Saunders, 1977.

11. American Psychiatric Association. Diagnostic and statistical manual of mental disorders. 3rd ed. Washington, DC: APA, 1982.

12. Kronfol Z, Turner R, Nasrallah H, Winokur G. Leukocyte regulation in depression and schizophrenia. Psychiatry Res 1984: 13:13-18.

13. Albrecht J, Holderman SH, Schlesser MA, Rush JA. A controlled study of cellular immune function of affective disorders before and during somatic therapy. Psychiatry Res 1985:15:185-193.

14. Syvalahti E, Eskola J, Ruuskanen O, Laine T. Nonsuppression of cortisol in depression and immune function. Prog Neuropsychopharmacol Biol Psychiatry 1985:9:413-422.

15. Fontana A, Storck U, Angst J, Dubs R, Abegg A, Grob PJ. An immunological basis for schizophrenia and affective disorders? Neuropsychobiology 1980:6:284-289. 
16. Legros S, Mendlewicz J, Wybran J. Immunoglobulins, autoantibodies and other serum protein fractions in psychiatric disorders. Eur Arch Psychiatry Neurol Sci 1985:235:9-11.

17. Carroll B, Curtis G, Davis BM, Mendel J, Sugerman A. Urinary free cortisol excretion in depression. Psychol Med 1976: 6:43-50.

18. Weiler J, Ballas Z, Needleman B, Hobbs M, Feldbush T. Complement fragments suppress lymphocyte immune responses. Immunol Today 1982:3:238-243.
Address

Ziad Kronfol, M.D

Associate Professor

Department of Psychiatry

University of Michigan Medical Center

UH-9C-9150-0120

1500 E. Medical Center Drive

Ann Arbor, MI 48109-0120

USA 